\section{Questión}

Periodismo / Comunicación ISSN 1669-6581

Sentidos y prácticas de sororidad en Facebook

Verónica Aráoz

Question/Cuestión, Vol. 2, N66, Agosto 2020

ISSNe 1669-6581

https://perio.unlp.edu.ar/ojs/index.php/question/index

IICom-FPyCS-UNLP

DOI: https//doi.org/10.24215/16696581e500

\title{
Sentidos y prácticas de sororidad en Facebook
}

\section{Senses and practices of sisterhood on Facebook}

Verónica Aráoz Instituto Académico Pedagógico de Ciencias Sociales, Universidad Nacional Villa María,

Consejo Interuniversitario Nacional, Universidad Católica de Santiago del Estero,

Argentina

veroaraoz90@gmail.com https://orcid.org/0000-0003-0500-699X

\section{Resumen}

En el siguiente escrito exploramos las prácticas y sentidos de sororidad en un grupo feminista de Facebook. Por lo que, comenzamos explicitando la 
categoría de sororidad para continuar con la descripción de los procesos de apropiación de las tecnologías digitales desde los planteos del Ciberfeminismo. Luego, retomamos el contexto de emergencia de un caso de estudio para analizar los sentidos y prácticas de sororidad que allí circulan. Finalmente reflexionamos sobre lo producido en este artículo.

\title{
Palabras clave
}

Sororidad, Facebook, Ciberfeminismo, Apropiación de TIC.

\begin{abstract}
In the following post we explore the practices and senses of sorority on Facebook. We begin by explaining the category of sisterhood to continue with the description of the processes of appropriation of digital technologies from the approaches of Cyberfeminism. Then, we return to the emergency context of a case study to analyze the senses and practices of sisterhood that circulate there. Finally we reflect on what has been produced on this article.
\end{abstract}

\section{Key Words}

Sisterhood, Facebook, Cyberfeminism, Appropiation of ICT

El presente escrito es una exploración y análisis sobre los sentidos y prácticas de sororidad del grupo de Facebook Feministas Trabajando en Córdoba, mujeres e identidades y sexualidades disidentes. A partir del estudio de caso identificamos prácticas de vinculación, de encuentro, de experiencias compartidas en el espacio digital que son leídas mediante la categoría de 
sororidad. Por lo tanto, analizamos una comunidad feminista que si bien desarrolla su entramado social en diversos lugares, en este artículo ponemos el foco en las relaciones que entabla dentro del medio digital. Además de constituirse explícitamente en una bolsa de trabajo para mujeres e identidades disidentes, este espacio digital es una apuesta simbólica para subvertir estereotipos de género, poner en debate temas de interés común, generar reconocimiento entre sus integrantes dentro de un orden patriarcal. Para adentrarnos a su estudio, establecemos una descripción del marco estructural social e histórico que enmarca la emergencia de la apropiación feminista de las redes sociales o herramientas digitales. A su vez, retomamos los aportes del ciberfeminismo como perspectiva que indaga acerca de la relación entre género y tecnología.

\section{Sororidad: una praxis política feminista}

En 2018, un año de variadas movilizaciones a favor de la legalización del aborto en el país y expresiones del movimiento feminista argentino, la palabra sororidad se impuso y afianzó dentro y fuera del colectivo. Inclusive ese mismo año la Real Academia Española la incluyó dentro del diccionario y distintos medios de comunicación publicaron que sororidad había sido la palabra más utilizada. Aunque ya en 2015 con la irrupción del \#NiUnaMenos se empezó a implementar para luchar de manera colectiva en contra de los femicidios y la violencia machista. Sin embargo, vale aclarar que dentro del campo de estudios de las teorías feministas es una categoría de análisis que tiene su trayectoria y que viene siendo discutida desde hace mucho tiempo.

Se entiende por sororidad una práctica política que con su implementación se propone erradicar la misoginia entre mujeres para empoderarse como 
colectivo frente a una sociedad machista y patriarcal. El término sororidad lo acuñó la feminista contemporánea y antropóloga mexicana Marcela Lagarde quien la define, en su escrito Pacto entre mujeres. Sororidad (2006), como: [...] una experiencia de las mujeres que conduce a la búsqueda de relaciones positivas y a la alianza existencial y política, cuerpo a cuerpo, subjetividad a subjetividad con otras mujeres, para contribuir con acciones específicas a la eliminación social de todas las formas de opresión y al apoyo mutuo para lograr el poderío genérico de todas y al empoderamiento vital de cada mujer (pp.126).

Lagarde (2006) hace referencia sobre el trabajo mancomunado entre mujeres para hacer frente a la desigualdad existente en relación a los varones. En este estudio, ampliamos la categoría incorporando al ejercicio de ésta práctica política a las identidades genéricas que no son mujeres cisi - trans, lesbianas y bisexuales - ya que también se encuentran subordinadas, dominadas 0 excluidas por la matriz heteronormativaii. Como menciona Lagarde (2006) se trata de una lucha colectiva. Basta con observar la trayectoria histórica del movimiento feministaiii para identificar que los derechos obtenidos se hicieron a través de la articulación de diversos y heterogéneos actores (Bustamante, 2010).

Encontramos antecedentes de la noción de sororidad en la formulación de la década del '70 de la estadounidense Kate Millet (1970) -perteneciente al feminismo radical de segunda olaiv_. Millet propuso el término en inglés sisterhood que traducido al español significa hermandad. Para entonces, los sentidos que se le atribuían eran los de constituir una unidad entre mujeres, no interesándose por las distinciones existentes entre ellas -como la raza, clase, etnia, edad, procedencia-. Por lo tanto, una de las formas de luchar 
contra la opresión de las mujeres y el patriarcado, según las feministas radicales, era la de entablar entre sí relaciones de solidaridad.

Luego, se retomó el concepto de sororidad en otras lenguas a partir de la raíz 'sor' del latín, así: en francés sororité, en italiano sorellanza y en castellano sororidad o soridad. Lagarde (2006) también vincula el concepto en cuestión con el de affidamento procedente del Colectivo de la Librería de Mujeres de Milán. Affidamento es definido en la Introducción Non credere di avere dei

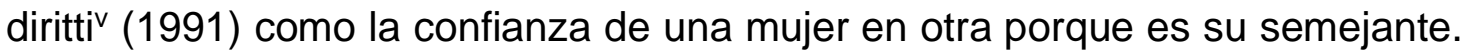
Es decir, da cuenta de una relación social entre mujeres, de una lucha política por liberarse del patriarcado. Por lo tanto, coincide con el significado atribuído a sisterhood por parte de las feministas radicales en donde el objetivo es formar un colectivo para ir en contra del patriarcado que oprime a las mujeres. A lo largo de este recorrido teórico-histórico, diversas y disímiles perspectivas feministas abordaron la idea de la alianza entre mujeres. Ocurre que el término sororidad cobra sentidos particulares según sea la concepción feminista que se trate -sea el feminismo de la igualdad o de la diferencia. Como indica Sonia Reverter Bañón (2003) los problemas que se quieren resolver son los mismos -la cuestión del poder, del trabajo, la separación de las esferas privada/pública, etc-, sobre todo el de erradicar un sistema que somete material y simbólicamente a las mujeres, pero cómo hacerlo es donde aparecen las diferencias (Reverter, 2003, pp. 42). Por ejemplo, desde la vertiente de las mujeres negras y lesbianas se subraya el hecho de que las mujeres no son un grupo homogéneo sino que dentro existe una gran diversidad.

Desde un feminismo latinoamericano, Lagarde (2006) entiende la sororidad como una alianza entre mujeres que comparten una condición común, la de 
opresión, pero donde se contemplan las diferencias que entre ellas pueda existir. "Aunque todas las mujeres viven en opresión genérica, la mayoría vive, además, la explotación de clase o de casta [...] Las diferencias entre las mujeres no son pequeñas. Integran espacios vitales diferenciados e impregnados de un sinfín de contradicciones y antagonismos" (Lagarde, 1987, pp. 13). Entonces, surge la pregunta sobre: ¿cómo es posible constituir un espacio común, una relación entre pares contemplando las experiencias particulares -posiciones disímiles en la estructura social-? La respuesta a ésta pregunta es que "[...] propiciamos la convivencia en la diversidad» (Lagarde, 2006, pp.130) en contra del pensamiento único, homogéneo y el dominio de una cultura jerárquica y excluyente que produce dominación.

Ahora bien, las diferencias con respecto a las mujeres son dos (Lagarde, 1987). Una tiene que ver con su relación con los varones -como el arquetipo de lo humano y paradigma patriarcal-. La otra se produce entre las mismas mujeres, se trata del distanciamiento y desconocimiento de lo que comparten en cuanto a condición genérica (Lagarde, 1987). Efectivamente, las mujeres son distintas a los varones, no en un sentido naturalista ya que se tratan de cuerpos históricamente construidos por la relación dialéctica entre la biología, sociedad y cultura. La diferencia radica en que las mujeres son producidas bajo la norma de la feminidad -donde adquieren y deben ejecutar determinados comportamientos, creencias, actitudes- (Lagarde, 2006). «La feminidad es la distinción cultural, históricamente determinada, que caracteriza a la mujer en sí misma y frente a la masculinidad del hombre" (Lagarde, 2006, p.6). El extrañamiento entre mujeres es la escisión del género que se produce cuando se erigen límites infranqueables imposibilitando el reconocimiento o la identificación entre sí. En este sentido, es preciso superar esas diferencias a 
través de su aceptación. Las mujeres habitan, aún siendo feministas, una estructura social donde impera el poder tradicional -jerárquico- y patriarcal, en donde son subjetivadas por dicha estructura social y sus significados circundantes -donde dominan las concepciones androcéntricas-.

Sin embargo, Lagarde (2006) no quiere decir que es determinante la estructura social sobre la construcción del sujeto femenino sino que hay un margen de acción, hay posibilidad(es) de resistencia frente a la sujeción. Esa resistencia se lleva a cabo a través del feminismo que "es la respuesta y la acción protagónica de las mujeres, a partir de su condición genérica, para subvertir el poder que las reproduce en la opresión» (Lagarde, 2006, p.6). A través de la sororidad como práctica política es posible desarticular la relación de enemistad histórica mujer-mujer y la opresión patriarcal entre los géneros.

\section{Apropiación de las TIC y ciberfeminismo}

De acuerdo a algunos estudios, la apropiación de las TIC refiere al proceso de interpretación material y simbólico que pone en relación los intereses identitarios, geográficos e históricos de una comunidad (Benítez Larghi Sebastián, 2009). Esta perspectiva se inserta en los estudios de carácter constructivista donde las tecnologías no son ajenas a las posibles valoraciones que los sujetos puedan hacer de acuerdo al contexto sociohistórico al cual pertenecen (Benítez, 2009). Si bien las tecnologías llevan impresas posibles interpretaciones, establecidas en su proceso de producción, no son determinantes las lecturas que de ellas puedan hacerse. Cuando los sujetos las utilizan les imprimen sus propias lecturas, significados, las dotan de sentidos y valoraciones correspondientes a su pertenencia social, género, edad, sexo, cultura (Benítez, 2009). 
En la Argentina de los últimos años se registran ejemplos de la apropiación de las TIC y plataformas infocomunicacionales por grupos feministas que fueron resonantes en la esfera pública. Entre ellos se puede mencionar el \#NiUnaMenos, la Campaña por el \#AbortoLegalYa, el \#8M (Laudano Claudia, 2018), entre otros. Diversas académicas señalan a este período como la cuarta ola feminista del movimiento feminista argentino que surge en 2015 con un momento de gran visibilidad y expansión de sus bases por la masiva convocatoria del primer $\mathrm{Ni}$ Una Menos en contra de la violencia machista y femicida (Elizalde Silvia, 2018; Hasan Fernández Valeria; 2019). En esta última etapa de visibilidad del movimiento feminista se registra un nuevo entramada de relaciones y vinculaciones entre las jóvenes e históricas del colectivo (Elizalde, 2018) y de prácticas como el ciberactivismo feminista (Laudano, 2018; Hasan, 2019). Sin embargo, existen instancias y antecedentes en nuestro país acerca de la apropiación de TIC por grupos feministas. Estas surgen con el auge y el desarrollo de Internet a fines de los '90 y principios del milenio. Entre las experiencias anteriores se registran en Argentina el caso de la lista de distribución de información por correo electrónico de la Red Informativa de Mujeres de Argentina, la lista de Jóvenes Feministas de colectivas de distintas provincias del país y el uso de blogs hasta 2011 cuando se produce una migración a plataformas infocomunicacionales como Facebook (Laudano, 2016; 2018).

Por otro lado, estas expresiones colectivas a través y mediante las TIC vienen siendo investigadas desde el ciberfeminismo. Esta corriente se inserta entre el entrecruzamiento de los Estudios de Género y Teorías Feministas con los Estudios Sociales de las Tecnologías (Ficoseco Verónica, 2016). Si bien en su interior contiene una diversidad y heterogeneidad de perspectivas, se ocupa 
de estudiar la relación entre las tecnologías y el género (Ficoseco, 2016). Las diferencias en los enfoques se deben a la concepción que se tenga de las tecnologías como señala Reverter (2001). En este caso, retomamos su categorización acerca de las distintas miradas que forman parte del ciberfeminismo. Según Reverter (2001) están las concepciones utópicas que conciben al ciberespacio como un lugar de liberación de estereotipos y de los sexos. Por otro lado, se encuentran las miradas distópicas sobre las tecnologías y el ciberespacio de corte determinista ya que las considera como herramientas de la dominación patriarcal. Por último, están las posturas que formulan una co-generación entre género y tecnologías. Es decir, ésta última supera las posturas dicotómicas o dualistas acerca de los aspectos positivos o negativos de las tecnologías y otorga una propuesta superadora. Esta perspectiva tiene su anclaje epistemológico en las teorías feministas de la performatividad (Reverter, 2001). La performatividad supone según Judith Butler (1990) la repetición estilizada de acciones y de las normas sociales, constituyendo la identidad de género del sujeto. Sin embargo, el sujeto no se encuentra totalmente determinado por las estructuras sociales ni tampoco está libre de elección desde un punto de vista voluntarista (Butler, 1990). Por lo tanto, existe la posibilidad de no repetir las normas, no hacerlo siempre de la misma manera o subvertirlas (Butler, 1990). En consecuencia, estos planteos posibilitan dejar de pensar desde un punto de vista determinista al género y a las tecnologías.

Dentro de ésta última vertiente encontramos los trabajos desarrollados desde el ciberfeminismo social (Ana de Miguel Montserrat Boix, 2002). Retomamos este enfoque ya que concibe al activismo feminista en red como la posibilidad de transformar la desigualdad que vivencian las mujeres (Boix, 2002) e 
identidades disidentes $\mathrm{o}$ no binarias. El ciberfeminismo social propone elaborar redes colaborativas que posibiliten compartir experiencias, identificar problemáticas y definir estrategias en conjunto para disputar al patriarcado (De Miguel, 2002; Perdomo, 2016). Conciben a Internet como un espacio de intercambio horizontal, que facilita la generación de redes o nexos para el activismo, para el pensamiento colectivo y el planteo de estrategias para avanzar hacia una democracia participativa (Boix, 2006). Por lo tanto, el activismo en red refiere a la acción política, colectiva y estratégica de las tecnologías para disputar las representaciones y símbolos culturales falocéntricos (Braidtotti, 1996; Boix, 2002). Sin embargo, es pertinente recuperar los planteos de Rosi Braidotti (1996) que señala un incremento de las desigualdades y asimetrías de género, clase, raza y culturales ${ }^{\mathrm{vi}}$ dentro del ciberespacio. Además, esta corriente identifica las diferencias entre los géneros en relación al acceso -a conocimientos y materiales- de herramientas digitales y en el diseño y programación de estos dispositivos (Braidotti, 1996; Boix, 2002).

\section{Contexto de emergencia de uso y apropiación de TIC}

En 2017 se crea en Córdoba un grupo de Facebook de feministas con el propósito de crear una bolsa de empleo. Exploramos este caso considerando, a modo de anticipación de sentido, que es un espacio digital donde se producen sentidos y prácticas de sororidad. Es decir, a través del grupo digital se crea un entramado común de relaciones de contención entre personas que comparten la opresión patriarcal. Por lo tanto, el grupo de facebook analizado constituye algo más que una bolsa de trabajo, de intercambios o compra y venta de productos y servicios. Se trata de un lugar de encuentro, de (de) 
construcción de los estereotipos de género, de puesta en debate de determinados temas de interés de la comunidad y de un espacio para compartir experiencias comunes al género. Por lo que si bien, a partir de la exploración etnográfica del grupo no se explicita o asume la categoría de sororidad éste se convierte en una categoría téorica que posibilita leer las acciones que se llevan a cabo. Como menciona Lagarde (2006) la sororidad es una acción, es resistencia, una respuesta política para subvertir la opresión patriarcal de los géneros.

El grupo de Feministas Trabajando Córdoba es un espacio que se origina como réplica de la organización creada en Buenos Aires en la misma plataforma infocomunicacional. Lo mismo ocurre en diversas provincias del país como el caso de Feministas Trabajando en Mendoza y Mujeres Trabajando Rosario. También se fueron creando otros espacios similares en las distintas localidades de Córdoba como el caso de Feministas Trabajando Punilla. Todos estos grupos de Facebook comparten un propósito que es el de generar un espacio de intercambios de trabajo, productos, servicios o una bolsa de empleo para identidades que no sean varones cis. A su vez, estos espacios presentan sus particularidades correspondientes a la comunidad que los integra, a otros propósitos que pueden guiar el grupo y al contexto local donde se sitúan.

El contexto histórico social bajo el cual surgen estos grupos nos permite explorar la razón por la cual se crearon estos espacios como bolsa de empleo para mujeres e identidades y sexualidades disidentes. Si bien desde el retorno a la democracia en 1983 se evidencian avances en la ampliación de los derechos para las mujeres e identidades disidentes y una revisión de sus condiciones de existencia (Barrancos, 2012), aún persiste la desigualdad y 
asimetrías entre los géneros. No es propósito de este escrito desarrollar un recorrido histórico acabado acerca de los logros y alcances obtenidos en materia de derechos para el colectivo de mujeres e identidades disidentes. Sí pretendemos exponer las condiciones estructurales que motivaron la emergencia de estos espacios digitales.

En la actualidad, la violencia machista, los femicidios ${ }^{\text {vii, }}$ transfemicidios y travesticidios continúan en aumento. La división sexual del trabajo viii persiste. En 2017, cuando se crea el grupo de Facebook Feministas Trabajando en Córdoba, la tasa de empleo formal para mujeres -de 43,1\%- es más baja que la de varones -de 66,3\%-, según datos de la Encuesta Permanente de Hogares (EPH) 2017 (Ministerio de Trabajo de la Nación, 2017). Son las mujeres las que se encargan del trabajo doméstico y de cuidado no remunerado. "Sostenemos nuestros hogares, teniendo como agravante la desigualdad salarial, que hace que las mujeres ganemos $27 \%$ menos que los hombres", decía el documento del Encuentro Nacional de Mujeres de 2017. Por otro lado, persiste la invisibilización de las identidades no binarias en la elaboración de datos oficiales. En 2012 la Organización Internacional del Trabajo (OIT) llevó a cabo un informe sobre la discriminación contra trabajadores lesbianas, gays, bisexuales y transexuales. Dicha publicación da cuenta de aquellos obstáculos para la incorporación de éstas identidades al empleo. En Argentina «[...] algunos/as empleadores/as esperan que las mujeres lesbianas afirmen su identidad femenina cambiando sus maneras y su forma de vestir» (OIT, 2012, p.1). También se indica que las mujeres trans encuentran como única estrategia de supervivencia el trabajo sexual. Por último, concluye que "[...] las personas LGBT encuestadas declararon no haber sido admitidas para un empleo debido a su orientación sexual [...]» (p. 
2). Por lo tanto, las identidades no cis género se encuentran vulnerables frente a una sociedad heteronormativa, aún con la Ley de Identidad de Género (26.743/12) promulgada en 2012 que posibilita tener en el documento nacional la identidad autopercibida.

Por otro lado, como indica la investigadora holandesa Van Dijck (2016), nos encontramos en una cultura de la conectividad. La penetración de los dispositivos digitales en la vida cotidiana de las personas ha llegado al punto que « [...] los adolescentes y los adultos jóvenes son incapaces de imaginar su vida social sin Facebook [...]» (Van Dijck, 2016, p.26), genera un contexto en donde los medios sociales intervienen en la configuración de las experiencias de las personas y de la identidad de las mismas. En el caso de Facebook $^{\text {ix }}$, como plataforma infocomunicacional sigue siendo un " [...] líder mundial indiscutible en el terreno de los sitios de redes sociales [...]” (Fumagalli et al., 2018, p. 5). En 2017, cuando se crea el grupo de Facebook Feministas Trabajando el Informe Técnico de Ciencia y Tecnología efectuado en el cuarto trimestre de ese año por el Instituto Nacional de Estadísticas y Censos (INDEC) denominado «Acceso y uso de tecnologías de la información y la comunicación» registraba que más del $60 \%$ de los hogares de sectores urbanizados tienen computadora y más del $75 \%$ cuentan con Internet. El mismo informe señalaba que en ese entonces 8 de cada 10 argentinos/as cuentan usan celular.

Por lo tanto, a partir de este marco estructural social e histórico es preciso reconocer la emergencia de una diversidad de grupos digitales feministas.

\section{Entablando redes feministas en Facebook}


Mediante la observación participante y etnografía virtual (Christine Hine, 2006) exploramos y analizamos los sentidos y prácticas de sororidad que se dan dentro del caso de estudio: Feministas Trabajando en Córdoba (mujeres e identidades y sexualidades disidentes) ${ }^{\mathrm{x}}$.En el presente trabajo retomamos como fuente de información el grupo de Facebook, contemplando los criterios de ética a la hora de investigar un grupo cerrado, y dos notas de medios locales realizadas a dos de sus administradoras ${ }^{\mathrm{x}}$. Pensamos a este espacio digital como a una comunidad virtual, es decir, un conjunto de personas que por intereses similares se vinculan en el ciberespacio o mediante herramientas digitales (Magallanes Udovicich, Mariana Loreta, 2015). En este caso se encuentra conformado solo por mujeres e identidades y sexualidades disidentes con el propósito de constituirse en una bolsa de empleo. Vale aclarar que esta comunidad tiene la particularidad de no solo encontrarse en y mediante las plataformas infocomunicacionales, también lo hacen en la Feria Feminista Trabajandoxii, creada en 2017 como emergente del espacio digital. Además, en la actualidad, desarrollaron una página web: «Feministas Trabajando es una plataforma gratuita de publicidad web que promociona los emprendimientos de Ixs compañerxs Usuarixs de La Grupa (es como la llaman quienes participan del espacio de Facebook)»xiii. Por lo tanto, existe una diversidad de espacios de encuentro de Feministas Trabajando en Córdoba. Si bien la comunidad inicia en el grupo de Facebook, luego se amplían los lugares de vinculación. Estos otros lugares son también contemplados en el presente análisis.

Por otro lado, las comunidades virtuales se caracterizan por su condición de mutación y dinamismo producto de sus intereses, orientaciones, las condiciones de las plataformas, entre otros aspectos (Magallanes, 2015). Por 
lo que la mutación del espacio digital y de las comunidades se constituye en una característica central ya que se encuentra en permanente construcción. Además, la plataforma infocomunicacional (Facebook) en la que se asienta la comunidad también varía sus normativas, posibilidades de uso, restricciones. Estos aspectos inciden en la apropiación y uso que de Facebook se haga. En este sentido, registramos que en sus comienzos este se constituía en un grupo privado, donde solo sus miembros pueden ver quiénes lo integran y qué publican, pero visible, se podía encontrar en las búsquedas de google o en Facebook. Esto se vio modificado en 2019 cuando el grupo fue cerrado y quienes administran abrieron uno nuevo modificando el espacio a uno privado y oculto, es decir, que solo sus miembros pueden encontrar el grupo en búsquedas de google o Facebook.

Por otro lado, el caso indagado se encuentra regulado por quienes la administran. En consecuencia las regulaciones que se fijen en el espacio responden a las dinámicas de interacción que se dan entre quienes participan. Ahora bien, quienes administran el espacio ocupan un papel central para el funcionamiento del grupo. En primer lugar son quienes filtran las publicaciones que no respondan a las pautas de convivencia, pre-establecidas y aceptadas por quienes integran el espacio. Por otro lado, son quienes materializan en las normas de convivencia demandas de diversa índole que se producen por quienes habitan el grupo. Esta delimitación del contenido a publicar se generó en los comienzos del espacio ya que se compartía información que no respondía al propósito de ser una bolsa de empleo. En ese entonces es que de este espacio se desprendieron y crearon nuevos grupos feministas orientados a otras actividades como: grupos feministas para la venta de indumentaria, grupos de feministas de alguna profesión liberal para compartir 
información común y grupos destinados a realizar denuncias, escraches 0 compartir experiencias comunes. Esta reproducción de espacios de comunidad feminista en Facebook puso en evidencia la necesidad de encontrarse, de conectar y de compartir espacios en común con quienes se identifican de ese modo.

En el caso del grupo que analizamos se evidencia que el objetivo central que guía el espacio es constituirse en una bolsa de empleo para mujeres e identidades y sexualidades disidentes. Una de las personas que administra el grupo considera que " [...] es una herramienta de encuentro y solidaridad entre compañeres, reconociendo que somos diferentes y nos atraviesan diversas problemáticas, pero que nos encuentran las ganas de resistir y combatir la opresión [...] » (Revista Educando, 2018). En este sentido, se lleva a cabo una acción colectiva con el propósito de subvertir ciertas condiciones estructurales que condicionan la existencia de las identidades que no son varones cis género. A su vez, se derivan de este espacio otros propósitos no explicitados. Tal es el caso de visibilizar que hay mujeres e identidades y sexualidades disidentes que realizan trabajos históricamente efectuados por varones cis género como: taxista/remisero, plomero, instalador de aires acondicionados, carpintero, electricista, entre otros. En este sentido como plantea Lagarde (2006) los feminismos buscan crear nuevas subjetividades e identidades por lo que la sororidad es central para la deconstrucción de los estereotipos de género. Con respecto a este aspecto también se observa en el uso del lenguaje no sexista y en la definición de feministas del grupo. Según una de las personas que administra el espacio: «El énfasis en 'feministas' y no 'mujeres' exclusivamente, es porque también entendemos que las categorías de género binarias (hombre-mujer) no son determinantes ni taxativas» (La 
Tinta, 2017). Con respecto al lenguaje que se utiliza dentro del espacio se observa además un código en común entre sus integrantes como la denominación del grupo de Facebook como "la grupa" o "la feria" para referirse a la Feria de Feministas Trabajando.

Dentro de este espacio se producen comportamientos particulares que denotan sororidad en términos de Lagarde (2006). Es el caso de las recomendaciones. Es decir, frente a una publicación sobre algún producto o servicio surge algún comentario de alguien que ya compró/probó lo ofrecido recomendándolo. Estas recomendaciones, el agradecimiento, el buen trato, el respeto y el consentimiento son pautas que se encuentran en las normas de convivencias y que reflejan el tipo de vinculaciones que se quieren generar. Vale recordar que en los inicios del grupo se produjo una ola de sorteos que comenzó con una publicación que ofrecía hacer un sorteo como agradecimiento por lo que le daba el espacio. Así se sumaron varias personas a hacer el mismo sorteo a modo de agradecimiento hasta que se volvió masivo, llegando a haber más de 1.000 participantes en cada uno (La Tinta, 2017). Al punto que se desvirtuó el propósito del espacio y tuvo que ser regulado por quienes administran limitando la publicación de más sorteos.

Como observamos, el grupo de Facebook se configuró en un espacio de encuentro, de vinculaciones entre quienes se autodefinen feministas, para compartir experiencias similares y para crear comunidad. A partir de este primer encuentro digital, en la "grupa", emergieron otros espacios como la Feria y actualmente la página Web donde se desarrolla esta comunidad feminista. 


\section{Reflexiones finales}

A modo de cierre, en primera instancia se puede decir que el espacio analizado no es solo una bolsa de empleo, de compraventa o de un intercambio comercial. Se trata también de una apuesta simbólica por establecer temas de agenda dentro de un grupo feminista y de generar rupturas con estereotipos de género. A su vez, es un espacio de vinculación, de encuentro, de intercambio de experiencias, de sororidad. Esta categoría conceptual, de sororidad, nos permite leer sentidos y prácticas que tienen como propósito generar alianzas, lazos, redes de soporte, reconocimiento entre mujeres e identidades y sexualidades disidentes para subvertir el orden patriarcal. En este sentido se podría continuar en el avance de la investigación indagando en quienes integran "la grupa" si perciben sororidad en el espacio, qué entienden por tal categoría.

Por otro lado, es preciso identificar que las acciones o estrategias colectivas que se llevan a cabo se ven motivadas por determinadas condiciones sociales. Es decir, en un contexto donde persiste la desigualdad de género, la división sexual del trabajo, la feminización de la pobreza, reunirse en un espacio es un modo de resistencia. A su vez, estas acciones de apropiación de las TIC o plataformas infocomunicacionales responde a una cultura de la conectividad y a un aumento de expresiones colectivas feministas en el ciberespacio como el \#NiUnaMenos, \#8M, \#AbortoLegalYa, entre otras ciberacciones. También responde al avance de normativas y leyes referidas a la ampliación de derechos para el colectivo de mujeres y disidencias que se produjeron desde la democracia a la actualidad. Además de las resistencias, luchas, movilizaciones gestadas por el movimiento feminista argentino y colectivo de mujeres. 
Finalmente, retomamos las críticas que desde el ciberfeminismo se advierten sobre la positividad de las TIC. Si bien las redes sociales son de fácil acceso, gratuitas y permiten la amplificación de las demandas a otros sectores sociales también persisten las brechas digitales. Se mencionan en plural porque no solo es la existencia aún de vastos sectores sociales sin accesos a las TIC. También es la falta de conocimiento sobre su uso y en este sentido se da una diferencia por sectores etarios. A su vez, el uso de las redes sociales se encuentra atravesado por la clase social, la procedencia, el sexo, la raza, la etnia.

' Cisgénero es la categoría que se utiliza para definir a una persona cuya identidad de género se corresponde con las expectativas sociales que se tienen de ella según el sexo asignado al nacer. Por ejemplo, una persona que nace con vulva, asignada como mujer al nacer y que se identifica como tal es considerada una mujer cisgénero.

ii Se entiende por matriz heteronormativa a un sistema de inteligibilidad de los cuerpos que permite a los sujetos relacionarse de una manera en particular. La relación dominante en este entramado es la heterosexualidad, invisibilizando otras formas de vincularse y, por ende, géneros. En tanto, que otros modos de comportarse y ser - lo que implica el género - quedan fuera de la norma, de "lo normal". En este sentido, la norma en este sistema es vivenciar un género en consecuencia al sexo asignado a la hora de nacer, es decir, si una persona fue asignada al nacer como varón su género es masculino (Butler, 1999).

iii En el presente proyecto se entiende al movimiento feminista como momentos de alianzas, acciones colectivas y políticas entre una multiplicidad de feminismos con divergentes agendas y posiciones (Bustamante, 2010).

iv En este caso retomamos la genealogía feminista que establece como olas a los momentos de fuerte visibilidad de las movilizaciones, luchas y de producciones teóricas por este colectivo (Triviño, 2019).

${ }^{v}$ Non credere di avere dei diritti traducido al español no creas tener derechos. 
vi Las plataformas infocomunicacionales, en su mayoría, son propiedad de corporaciones norteamericanas. Esto se traduce en marcas culturales que quedan impresas en el diseño y programación de las herramientas digitales (Braidotti, 1996).

vii Cada 30 horas una mujer es asesinada, según el 10 Índice Nacional de Violencia Machista publicado en 2016 e impulsado por el colectivo Ni una menos.

viii Se retoman lo planteos de Silvia Federici (2013) sobre la división sexual del trabajo. La autora plantea que las mujeres fueron asignadas al trabajo reproductivo no asalariado, mientras los varones al trabajo productivo remunerado.

ix Esta red social surge en 2004 fundada por Mark Zuckerberg con el nombre de «TheFacebook» con el propósito de conectar a usuarios que tuvieran una casilla de email de la Universidad de Harvard. Luego, al tener éxito en tan poco tiempo se extendió su uso -en 2005- a las demás instituciones universitarias de Estados Unidos y se transformó en Facebook. Ocurrido esto, el fundador decidió expandir su uso, no limitándolo al espacio universitario y educativo, posibilitando su acceso a cualquier persona mayor de 13 años que tuviera una cuenta de email (Fumagalli et al., 2018).

x Se contempló como criterio ético de exploración del caso de estudio, no reproducir publicaciones, exponer nombres de quienes integran el grupo o administran para resguardar la identidad de quienes forman parte de dicho espacio. En este sentido, para acceder a la información se recolectaron notas periodísticas de medios de comunicación locales realizadas a quienes administran el grupo que se indaga. También como investigadora y miembro del grupo de Facebook analizado realicé una observación participante dentro del campo de estudio como un nativo (Guber, 2001). Para ello, previamente a desarrollar la investigación me puse en contacto con quienes administran el grupo a quienes comenté mi intención de desarrollar una investigación y quienes otorgaron permiso.

${ }^{x i}$ En este escrito retomamos solo dos notas acerca del grupo a indagar ya que tienen la particularidad de ser entrevistas a dos de quienes administran o administraron el espacio.

xii Cuenta con su propia página de Facebook para difundir información únicamente acerca de la Feria. En este caso recuperamos la reseña de la página web oficial de Feministas Trabajando. 


\section{Bibliografía}

Acceso y uso de tecnologías de la información y la comunicación (2017). Instituto Nacional de Estadísticas y Censos (INDEC). https://www.indec.gob.ar/indec/web/Nivel3-Tema-4-26 Alerta Feministas Trabajando (3 de agosto de 2018). La Tinta. Recuperado de https://latinta.com.ar/2017/08/alerta-feministas-trabajando/

Barrancos, D. (2012). Mujeres en la sociedad argentina. Una historia de cinco siglos. Buenos Aires: Ed. Sudamericana.

Braidotti, R. (1996): Cyberfeminism with a difference. Traducción castellana, Un ciberfeminismo diferente. Carolina Díaz. E-mujeres.net. Recuperado de https://e-mujeres.net/rosi-braidotti-un-ciberfeminismo-diferente/

Benítez Larghi, S. (2009). Los sentidos de la comunicación en la apropiación de las TIC por las organizaciones de trabajadores desocupados. Question/Cuestión, 1(24). Recuperado de https://perio.unlp.edu.ar/ojs/index.php/question/article/view/864 Boix, Montserrat, A. (2002a). Feminismos, comunicación y tecnologías de la información. Mujeres en Red. Recuperado de http://www.mujeresenred.net/m boix-feminismo y comunicacion.html (2002b). Los géneros de la red: los ciberfeminismos. Mujeres en Red. Recuperado en http://www.mujeresenred.net/spip.php?article297

Butler, J. (1999). El género en disputa. El feminismo y la subversión de la identidad. España: Paidós. 
Butler, J., \& Lourties, M. (1998). Actos performativos y constitución del género: un ensayo sobre fenomenología y teoría feminista. Revista Debate Feminista, 18.https://doi.org/https://doi.org/10.22201/cieg.2594066xe.1998.18.5 26

Elizalde, S. (2018). Hijas, hermanas, nietas: genealogías políticas en el activismo de género de las jóvenes. Revista Ensambles (4). Recuperado de http://www.revistaensambles.com.ar/ojs-

\subsection{1/index.php/ensambles/article/view/149}

Encuesta Permanente de Hogares (EPH) (2017). Instituto Nacional de $\begin{array}{llll}\text { Estadísticas } y \text { Censos (INDEC) Recuperado de } & \text { y }\end{array}$ https://www.indec.gob.ar/indec/web/Institucional-Indec-BasesDeDatos Feministas Trabajando (26 de noviembre de 2018). Educando del IPEM 207 Eduardo Requena. Recuperado de http://www.requena.com.ar/?p=1300 Federici, S. (2013). Revolución en punto cero. Trabajo doméstico, reproducción $y$ luchas feministas. Traficante de Sueños. Recuperado de https://www.traficantes.net/sites/default/files/pdfs/Revolucion\%20en\%20punto\% 20cero-TdS.pdf

Ficoseco, V. (2016) Mujeres y Tecnologías Digitales. Antecedentes del Campo de los Estudios de Género para el análisis de esta confluencia. Revista Extraprensa, 9(2), 87-98. https://doi.org/10.11606/extraprensa2016.107719 Fumagalli, A (et.al) (2018). El trabajo (labour) digital en la economía de plataforma: el caso de Facebook. Hipertextos 6(9). Recuperado de http://revistahipertextos.org/wp-content/uploads/2015/12/1.-Fumagalli-et-al.pdf 
Guber, R. (2001). La etnografía. Método, campo y reflexividad. Enciclopedia Latinoamericana de Sociocultura y Comunicación. Bogotá: Grupo Editorial, Norma.

Hine, C. (2004). Etnografía virtual. Barcelona.UOC.

La discriminación en el trabajo por motivos de orientación sexual e identidad de género: Resultados del proyecto PRIDE de la OIT (2012). Servicio de Género, Igualdad y Diversidad (GED), Departamento de Condiciones de Trabajo e Igualdad.

Lagarde, M. (1987). Enemistad y sororidad: hacia una nueva cultura feminista. E-mujeres.net. Recuperado de https://e-mujeres.net/wpcontent/uploads/2016/08/Enemistad-y-sororidad.pdf

(2006). Pacto entre mujeres. Sororidad. E-mujeres.net. Recuperado de https://e-mujeres.net/pacto-entre-mujeres-sororidad/ Laudano, C. (2018). Acerca de la apropiación feminista de TICS. En Chaher, S. (comp.) Argentina: medios de comunicación y género ¿hemos cumplido con la plataforma de acción de Beijing? Ciudad Autónoma de Buenos Aires, Argentina. Recuperado de https://diariofemenino.com.ar/df/wpcontent/uploads/2019/03/Argentina-CSW2018comunicaci\%C3\%B3n g\%C3\%A9nero-FINAL2.pdf Magallanes Udovicich, M. L. (2015). Producción y uso de conocimiento en comunidades virtuales (Tesis doctoral). Recuperado de http://sedici.unlp.edu.ar/handle/10915/49838

Millet, K. (1970). Política Sexual. Traducción castellana, Sexual Politics. Ana María Bravo García. Ediciones Cátedra. 
Pobreza y represión: El ajuste golpea a sectores populares y sobre todo perjudica a las mujeres. (14 de octubre de 2017). Diario Norte. Recuperado de https://www.diarionorte.com/article/158328/pobreza-y-represion-el-ajustegolpea-a-sectores-populares-y-sobre-todo-perjudica-a-las-mujeresReverter Bañón, S. (2001). Reflexiones en torno al Ciberfeminismo. Asparkía: investigación feminista, (12). Recuperado de http://www.erevistes.uji.es/index.php/asparkia/article/view/883/0 (2013). Ciberfeminismo: de virtual a político .

TeknoKultura, Revista de Cultura Digital y Movimientos Sociales 10(2), 451461. Recuperado de https://revistas.ucm.es/index.php/TEKN/article/view/51905 Triviño, B. R. (2019). Feminismos: Antología de textos feministas para uso de las nuevas generaciones, y de las que no lo son tanto. Madrid. Los Libros de la Catarata.

Van Dijck, J. (2016) La cultura de la conectividad: Una historia crítica de las redes sociales.

Buenos Aires. Recuperado de http://catedradatos.com.ar/media/La-cultura-dela-conectividad -Jose-Van-Dijck.pdf 